\title{
The Protective Effects of Enalapril Maleate and Folic Acid Tablets against Contrast-Induced Nephropathy in Diabetic Rats
}

\author{
Jiantong Hou, Gaoliang Yan, Bo Liu, Boqian Zhu, Yong Qiao, Dong Wang, \\ Ruifeng Li, Erfei Luo, and Chengchun Tang $\mathbb{C}$ \\ Department of Cardiology, Zhongda Hospital of Southeast University Medical School, Nanjing 210009, China
}

Correspondence should be addressed to Chengchun Tang; tangchengchun@medmail.com.cn

Received 2 June 2017; Accepted 9 January 2018; Published 7 February 2018

Academic Editor: Enzo Terreno

Copyright (C) 2018 Jiantong Hou et al. This is an open access article distributed under the Creative Commons Attribution License, which permits unrestricted use, distribution, and reproduction in any medium, provided the original work is properly cited.

\begin{abstract}
Background. Renal vasoconstriction, oxidative stress, endothelial dysfunction, and apoptosis are the major causes of contrastinduced nephropathy (CIN). The aim of this study was to evaluate the protective effects of enalapril maleate and folic acid tablets on CIN in diabetic rats. Methods. Thirty-two Sprague-Dawley rats were divided into four groups: CIN (C), CIN + enalapril maleate (CE), CIN + folic acid (CF), and CIN + enalapril maleate and folic acid tablets (CEF). CE, CF, and CEF rats were treated orally with enalapril maleate, folic acid, or enalapril maleate and folic acid tablets, respectively, for 5 days. CIN was induced in all groups followed by analyzed biochemical parameters, oxidative stress markers, endothelial dysfunction parameters, renal histopathology, and TUNEL staining. Results. Serum creatinine, blood urea nitrogen, and malondialdehyde levels were lower in the CEF group than in the $\mathrm{C}$ group. Homocysteine, superoxide dismutase, glutathione peroxidase, and nitric oxide levels were higher in the CEF group than in the $\mathrm{C}$ group. Histopathology scores and percentage of apoptotic kidney cells in the CEF group were significantly decreased compared with those in the $\mathrm{C}$ group. Conclusions. These results suggest that enalapril maleate and folic acid tablets have a protective effect against $\mathrm{CIN}$ in diabetic rats.
\end{abstract}

\section{Introduction}

Contrast-induced nephropathy $(\mathrm{CIN})$ is a relatively common and serious complication that can occur after the intravascular administration of iodinated radiographic contrast medium. CIN is defined as an increase in serum creatinine (Scr) of more than $25 \%$ or $44.2 \mathrm{mmol} / \mathrm{L}^{-1} 48$ to 72 hours after contrast medium administration without evidence of other causes [1]. Among all causes of hospital-acquired acute kidney injury (AKI), CIN is the third most common and accounts for $11 \%$ of all cases [2]. In the general population, the incidence of CIN is estimated to be almost $2 \%$ [3]. However, the incidence of CIN in patients with diabetes mellitus $(\mathrm{DM})$ and chronic kidney disease (CKD) can approach $50 \%[4,5]$. In addition, DM can significantly enhance the occurrence of CIN in patients with renal insufficiency [6]. This life-threatening disease causes prolonged hospitalization and increases the incidence of cardiovascular events and mortality [7]. However, there are still no effective measures to prevent the occurrence of this serious disease. Therefore, the search for more reliable and effective way to treat CIN is critical for patients at high risk of developing CIN.

The pathogenesis of CIN has not been completely explained by a single mechanism, but the development of CIN occurs through a combination of various mechanisms, including acute renal vasoconstriction, contrast medium cytotoxicity to renal tubular cells, oxidative stress, endothelial dysfunction, and apoptosis [8-10]. Homocysteine (Hcy), a sulfhydryl-containing amino acid, is as an intermediate product in the normal biosynthetic pathway of the amino acids such as methionine and cysteine [11]. Hcy levels are significantly elevated in patients with CKD [12]. Hyperhomocysteinemia is directly related to endothelial dysfunction and apoptosis and enhances the occurrence of oxidative stress [13-15]. Enalapril maleate and folic acid tablets are a new compound preparation containing both enalapril and folic 
acid and are the first approved treatment for hypertension associated with elevated Hcy [16]. Folic acid plays a key role in reducing oxidative stress, improving endothelial function, and preventing apoptosis by reducing Hcy. Moreover, enalapril, a well-known antihypertensive drug, can prevent and treat CIN progression by promoting renal vasodilation [17].

However, no relevant report is currently available regarding whether enalapril maleate and folic acid tablets have a protective effect against CIN. We hypothesized that enalapril maleate and folic acid tablets could prevent CIN due to their renal vasodilation, antioxidative, endothelial-functionpromoting, and antiapoptotic effects. In this study, our aim was to investigate the renal protective effects of enalapril maleate and folic acid tablets against CIN in diabetic rats by analyzing biochemical parameters, oxidative stress markers, endothelial dysfunction parameters, histopathological staining, and apoptosis.

\section{Materials and Methods}

2.1. Animals. Male Sprague-Dawley rats $(300 \pm 10 \mathrm{~g})$ were obtained from the Southeast University Animal Center. The animals were housed in a vivarium under controlled photocycle $\left(12 \mathrm{~h}\right.$ light $/ 12 \mathrm{~h}$ dark) and temperature $\left(22-25^{\circ} \mathrm{C}\right)$ conditions with free access to food and water. Our study was approved by the Ethics Review Board for Animal Studies at the Institute of Southeast University, Nanjing, China.

2.2. Induction of Diabetes. Twelve hours after fasting, streptozotocin $(60 \mathrm{mg} / \mathrm{kg}$; Sigma, St. Louis, MO) was injected intraperitoneally to establish early diabetes mellitus. Blood glucose concentrations were determined after three days, and rats with blood glucose levels over $300 \mathrm{mg} / \mathrm{dl}$ were considered to have diabetes mellitus. Glucose levels were tested in rats every day.

2.3. Grouping and Drug Intervention. Thirty-two rats were divided into four groups of eight rats: (1) CIN (C), (2) $\mathrm{CIN}+$ enalapril maleate (CE), (3) CIN + folic acid (CF), and (4) CIN + enalapril maleate and folic acid (CEF). Rats in the $\mathrm{C}, \mathrm{CE}, \mathrm{CF}$, and $\mathrm{CEF}$ groups received saline, enalapril maleate (Yangzijiang Pharmaceuticals, Jiangsu, China) at $30 \mathrm{mg} / \mathrm{kg} /$ day, folic acid (Changzhou Pharmaceutical, Jiangsu, China) at $2.4 \mathrm{mg} / \mathrm{kg} / \mathrm{day}$, or enalapril maleate and folic acid tablets (Shenzhen AUSA, Guangdong, China) at $30 \mathrm{mg} / 2.4 \mathrm{mg} / \mathrm{kg} /$ day, respectively, for 5 consecutive days before CIN was induced.

2.4. Induction of CIN. CIN was induced by sequential tail vein administration of indomethacin $(10 \mathrm{mg} / \mathrm{kg})$, L-NAME $(10 \mathrm{mg} / \mathrm{kg}$, twice at 15 and $30 \mathrm{~min})$, and loperamide $(10 \mathrm{ml} / \mathrm{kg}$, Bayer, Germany) in rats. Scr concentration was determined according to the CIN definition to evaluate whether the CIN model was successful. The rats were sacrificed 3 days after the induction of CIN. Serum was separated from blood to determine biochemical and endothelial dysfunction parameters. Renal tissues were removed to evaluate oxidative stress markers, histopathology, and apoptosis.
2.5. Biochemical Parameters. Blood samples were removed by heart puncture and separated to obtain serum. Scr, blood urea nitrogen (BUN), and Hcy were measured using an autobiochemistry analyzer AU5800 (Beckman Coulter, USA) within $24 \mathrm{~h}$.

2.6. Oxidative Stress Markers. Superoxide dismutase (SOD), malondialdehyde (MDA), and glutathione peroxidase (GSHPX) levels were determined using experimental kits (Nanjing Jiancheng, China) in renal tissue homogenates.

2.7. Endothelial Dysfunction Parameters. Concentrations of serum nitric oxide (NO) and vascular endothelial growth factor (VEGF) were analyzed using commercial kits (Nanjing, Jiancheng, China) according to the manufacturer's instructions.

2.8. Renal Histopathology. Kidneys were removed from rats and placed in $4 \%$ paraformaldehyde for $24 \mathrm{~h}$, followed by paraffin embedding and sectioning. Renal specimens were then stained with hematoxylin and eosin. We used a renal injury scoring system to assess the degree of kidney damage. Criteria for renal injury were as follows: 0 , no injury; 1 , minimal tubular epithelial cell edema; 2, hemorrhage and moderate tubular epithelial cell edema; 3 , moderate hemorrhage and moderate tubular epithelial cell edema; 4 , severe tubular epithelial cell edema and formation of necrosis spots; and 5, intratubular cast presence, severe tubular architecture alteration, and severe tubular necrosis [18]. Evaluations were performed in a blinded manner.

2.9. TUNEL Staining. DNA fragmentation in kidney apoptotic cells was determined by TUNEL staining according to the manufacturer's instructions (Roche, Mannheim, Germany). Briefly, kidney samples were paraffin-embedded and cut into $3 \mu \mathrm{m}$ sections followed by deparaffinization and rehydration. Kidney sections were pretreated with proteinase-K and then incubated with TUNEL reaction mixture for $1 \mathrm{~h}$ at $37^{\circ} \mathrm{C}$. The number of TUNEL-positive cells was counted in 10 randomly selected nonoverlapping fields in each kidney sample under 400x magnification.

\section{Statistical Analysis}

The data are expressed as the mean \pm standard deviation. Data were analyzed using one-way ANOVA followed by Tukey's Multiple Comparison Test using SPSS17.0 (SPSS Inc., Chicago IL, USA). $P$ values less than 0.05 were considered statistically significant.

\section{Results}

4.1. Biochemical Parameters. Scr and BUN levels were significantly lower in the CE, CF, and CEF groups than in the $\mathrm{C}$ group $(P<0.01)$. Treatment with folic acid or enalapril maleate combined with folic acid significantly decreased serum Hcy levels (CF and CEF versus C, $P<0.01$ ). In addition, serum Hcy levels were lower in the CEF group than in the CE group $(P<0.05)$ (Figure 1). 


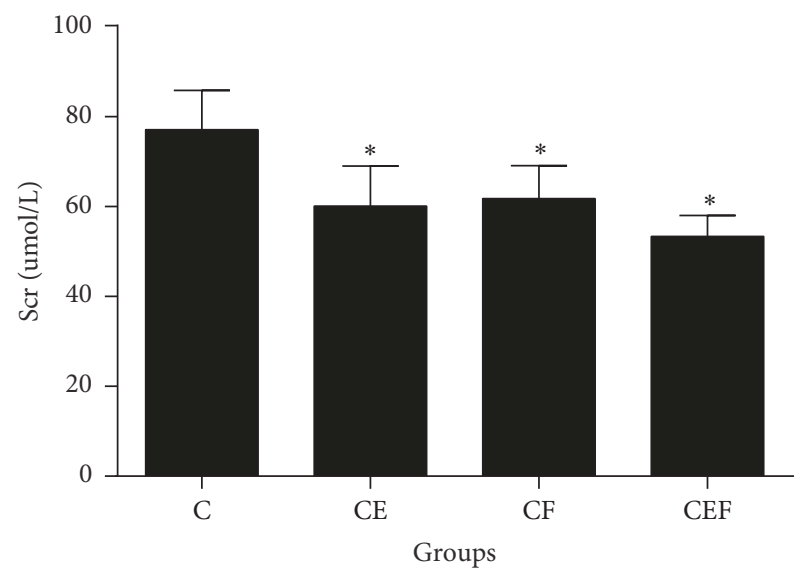

(a)

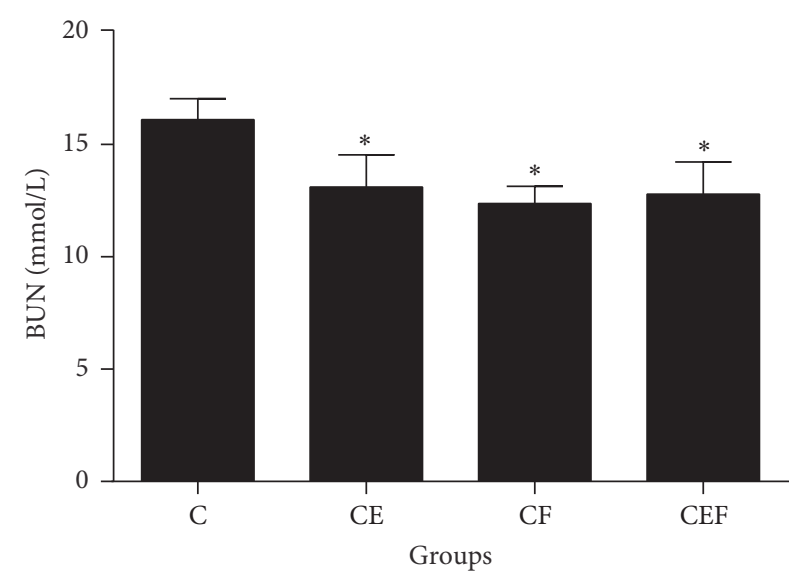

(b)

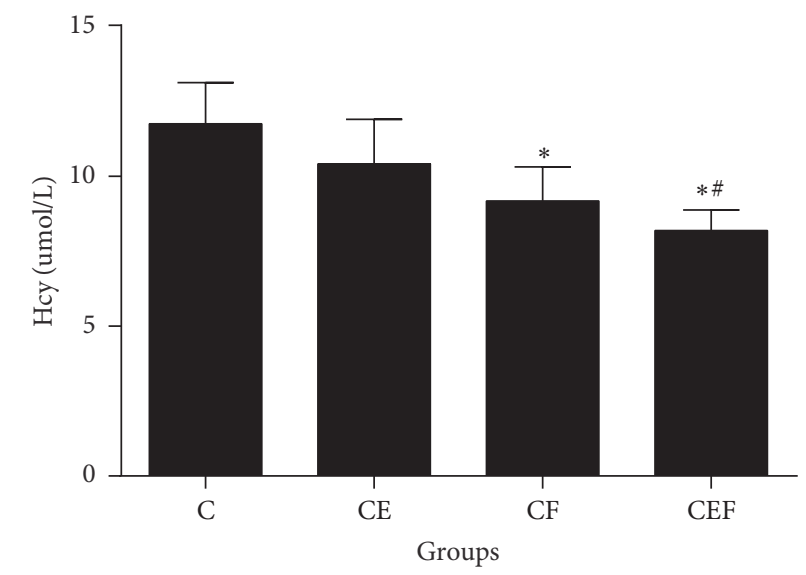

(c)

FIGURE 1: C: contrast-induced nephropathy; CE, contrast-induced nephropathy with enalapril maleate treatment; CF, contrast-induced nephropathy with folic acid treatment; $\mathrm{CEF}$, contrast-induced nephropathy with enalapril maleate and folic acid treatment. Data are presented as the means $\pm \mathrm{SD}(n=8) .{ }^{*} P<0.01$ versus $C ;{ }^{\#} P<0.05$ versus $\mathrm{CE}$.

4.2. Oxidative Stress Markers. As shown in Figure 2, kidney tissue homogenate SOD levels were higher in the CEF group than in the $\mathrm{C}$ and $\mathrm{CF}$ groups $(P<0.05)$. MDA levels were lower in the $\mathrm{CE}$ and CEF groups than in the $\mathrm{C}$ and $\mathrm{CF}$ groups $(P<0.01$ and $P<0.05$, respectively). GSH-PX levels were higher in the $\mathrm{CE}$ and CEF groups than in the $\mathrm{C}$ group $(P<$ 0.05 and $P<0.01$, respectively).

4.3. Endothelial Dysfunction Parameters. As shown in Figure 3, serum NO levels were notably increased in the CEF group compared with those in the $\mathrm{C}$ group $(P<0.05)$. However, there were no statistically significant differences in serum VEGF between the CE, CF, CEF, and C groups $(P>$ 0.05).

4.4. Renal Histopathology. Figure 4 shows that there were no significant differences in kidney injury scores between the $\mathrm{CE}, \mathrm{CF}$, and $\mathrm{C}$ groups $(P>0.05)$. However, the renal histopathology score in the CEF group was lower than that in the $\mathrm{C}$ group $(P<0.05)$. This suggests that enalapril maleate and folic acid tablets could significantly decrease kidney injury due to contrast medium.

4.5. TUNEL Staining. As shown in Figure 5, TUNEL staining showed that contrast injection can lead to elevated apoptosis in diabetic rats. The percentage of kidney apoptotic cells was significantly lower in the CEF group than in the $\mathrm{C}$ group $(P<0.01)$. In addition, the percentage of kidney apoptotic cells was also decreased in the CEF group compared with those in the CE and CF groups $(P<0.05$ and $P<0.01$, respectively). This suggests that enalapril maleate and folic acid tablets could exert an antiapoptotic effect in CIN rats.

\section{Discussion}

CIN has become an increasingly serious iatrogenic complication in clinical practice due to the high morbidity and mortality rates. Although significant efforts to develop prevention and treatment strategies to reduce the incidence and severity of CIN have been made, the results are still unsatisfactory and 


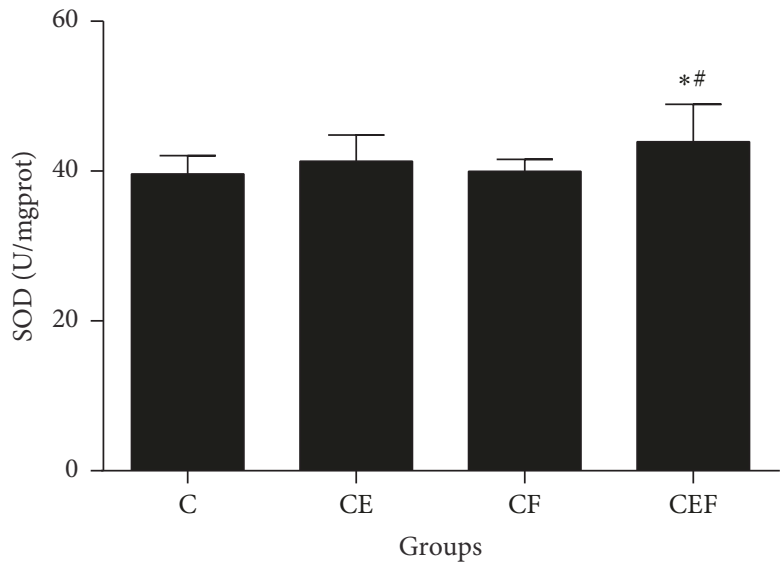

(a)

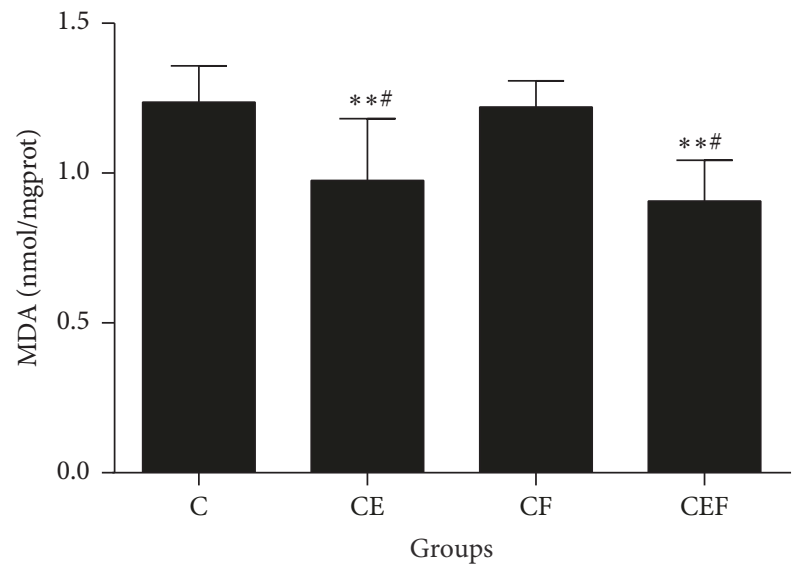

(b)

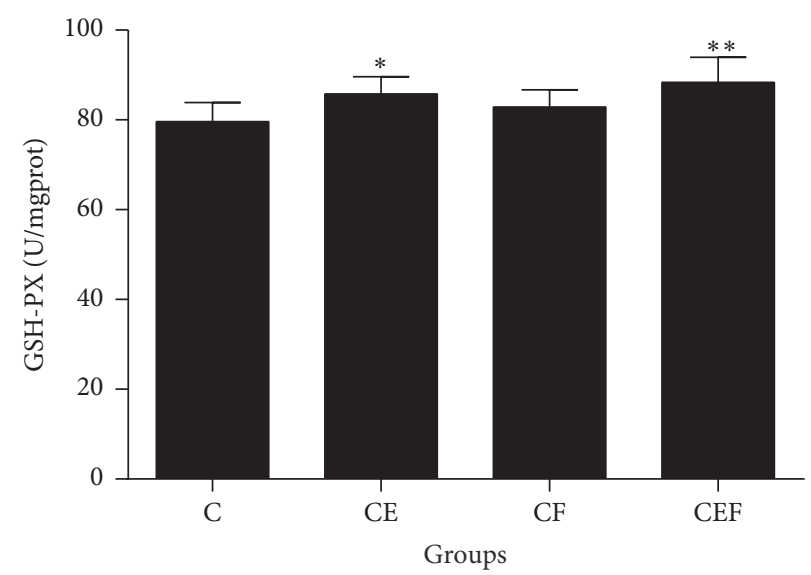

(c)

Figure 2: C: contrast-induced nephropathy; CE, contrast-induced nephropathy with enalapril maleate treatment; CF, contrast-induced nephropathy with folic acid treatment; $\mathrm{CEF}$, contrast-induced nephropathy with enalapril maleate and folic acid treatment. Data are presented as the means $\pm \operatorname{SD}(n=8) .{ }^{*} P<0.05$ versus $C,{ }^{* *} P<0.01$ versus $C$, and ${ }^{*} P<0.05$ versus $C F$.

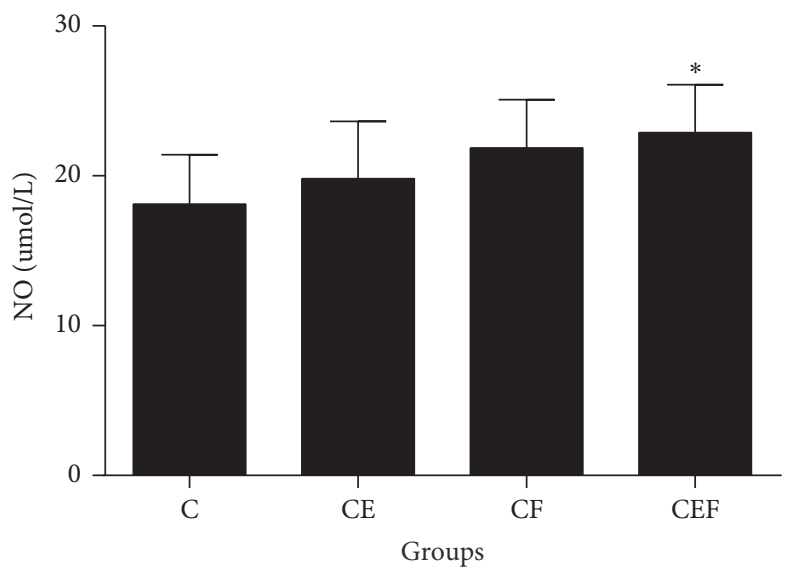

(a)

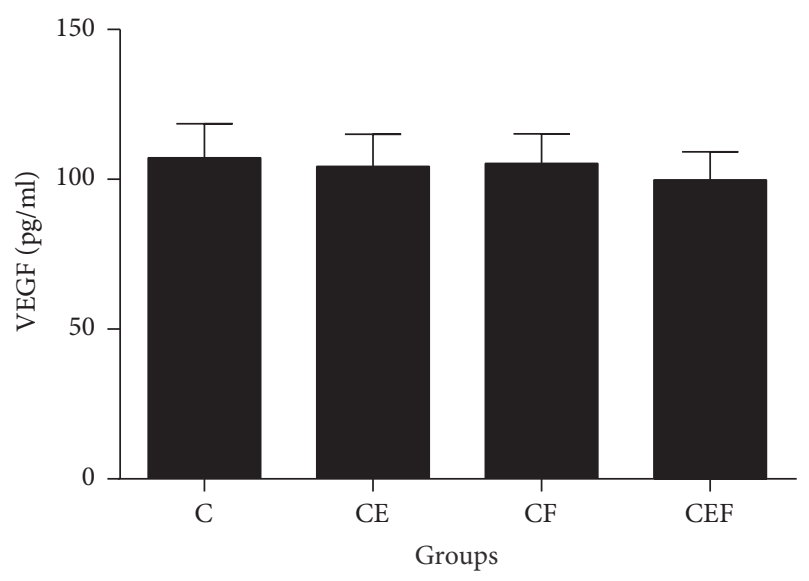

(b)

FIGURE 3: C: contrast-induced nephropathy; CE, contrast-induced nephropathy with enalapril maleate treatment; CF, contrast-induced nephropathy with folic acid treatment; $\mathrm{CEF}$, contrast-induced nephropathy with enalapril maleate and folic acid treatment. Data are presented as the means $\pm \mathrm{SD}(n=8) .{ }^{*} P<0.05$ versus $\mathrm{C}$. 
C

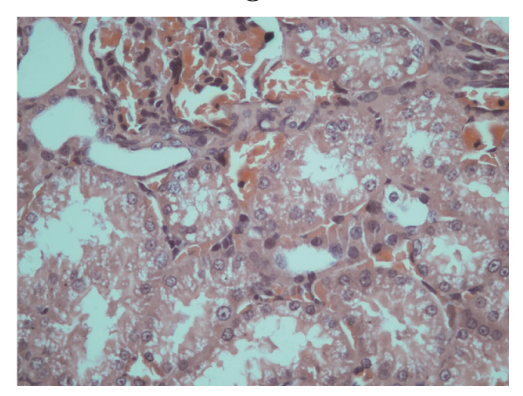

CF
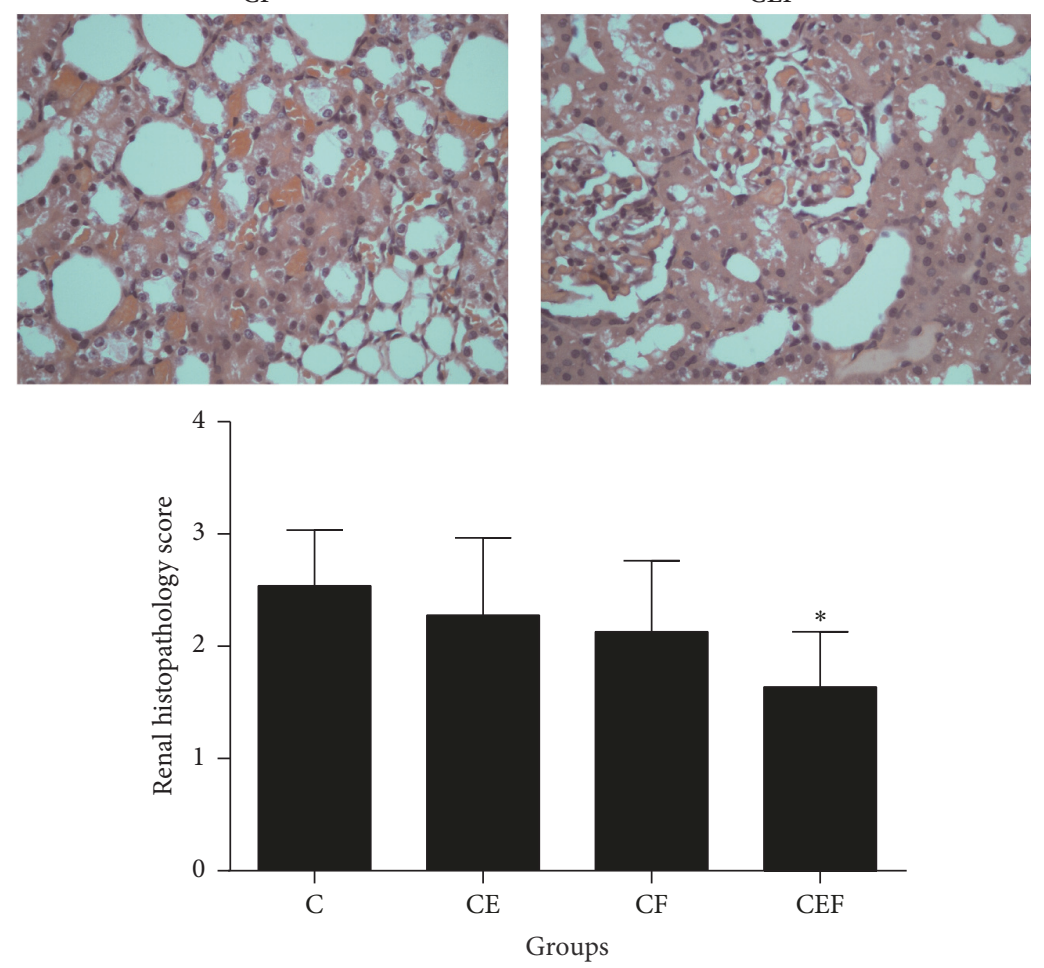

$\mathrm{CE}$

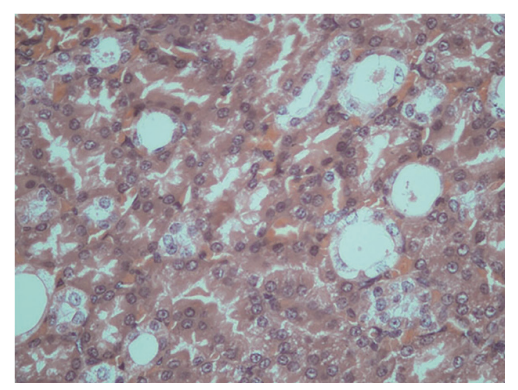

CEF

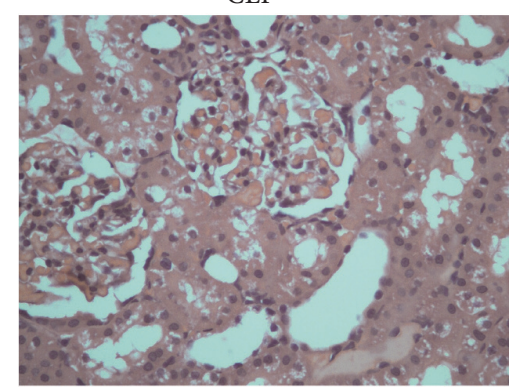

FIGURE 4: C: contrast-induced nephropathy; CE, contrast-induced nephropathy with enalapril maleate treatment; CF, contrast-induced nephropathy with folic acid treatment; $\mathrm{CEF}$, contrast-induced nephropathy with enalapril maleate and folic acid treatment. Data are presented as the means $\pm \mathrm{SD}(n=8) .{ }^{*} P<0.05$ versus $\mathrm{C}$.

inconsistent $[19,20]$. Therefore, new methods of prevention and treatment for CIN could have significant clinical value. In this study, we demonstrated that enalapril maleate and folic acid tablets can decrease the incidence of CIN in diabetic rats. Our data indicated that pretreatment with enalapril maleate and folic acid tablets could significantly decrease renal injury biomarkers, including Scr and BUN, as well as Hcy. In addition, enalapril maleate and folic acid tablets ameliorated renal function, oxidative stress, endothelial dysfunction, kidney histopathological injury, and apoptosis in CIN rats.

Although the pathogenesis of CIN is not completely understood, CIN is mainly due to acute renal vasoconstriction, oxidative stress, endothelial dysfunction, and apoptosis [8-10]. A previous study also showed that contrast medium can lead to increased release of adenosine, endothelin, and other renal vasoconstrictors, which causes acute renal vasoconstriction and subsequent ischemia-reperfusion injury [21,
22]. Enalapril, an angiotensin-converting enzyme inhibitor (ACEI), can inhibit the synthesis of angiotensin II resulting in vasodilation. It can also enhance the vasodilatory effect of bradykinin to promote vasodilatation further [23]. In this study, we showed that pretreatment with enalapril maleate can significantly decrease Scr and BUN levels. Therefore, we hypothesized that enalapril may improve renal function by promoting vasodilation.

Studies have shown that oxidative stress and the production of reactive oxygen species (ROS) are also important mechanisms in the occurrence and development of CIN [24]. Several studies have shown that angiotensin II promotes the production of ROS by enhancing the synthesis of growth factors and profibrotic cytokines $[25,26]$. Enalapril maleate and folic acid tablets may exert an antioxidant effect by inhibiting the synthesis of angiotensin II. Increased serum $\mathrm{Hcy}$ is closely related to the occurrence of oxidative stress in 
C

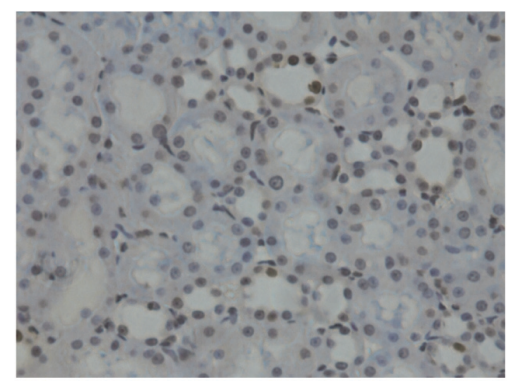

CF
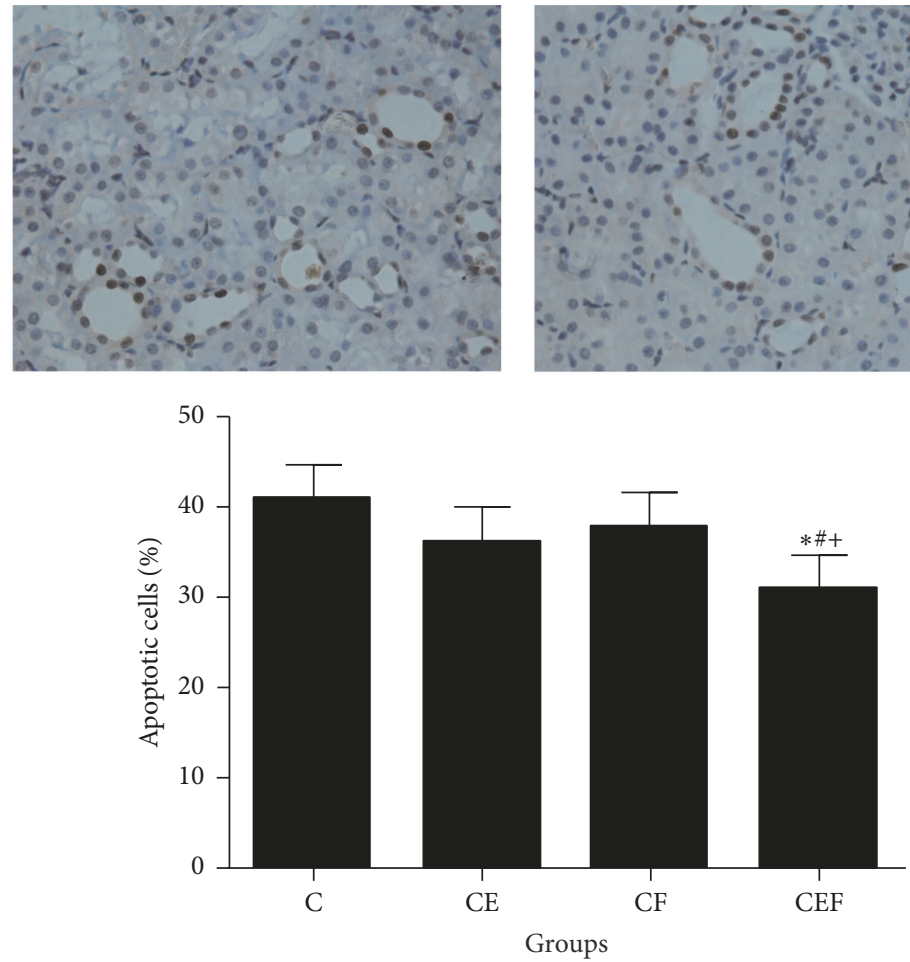

CE

CEF
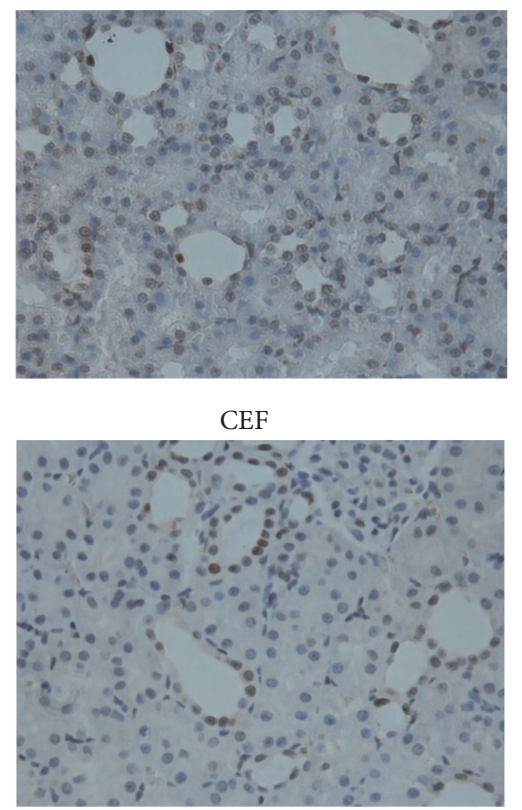

\section{政}


reticulum stress and apoptosis in pancreatic islet $\beta$ cells [38]. This may be related to folic acid deficiency leading to elevated Hcy levels. A previous study showed that Hcyinduced apoptosis of endothelial cells may occur through the accumulation of ROS and the inhibition of NO synthesis $[15,39,40]$. In the present study, we observed that enalapril maleate and folic acid tablets could significantly reduce the percentage of kidney apoptotic cells in CIN rats. Enalapril maleate and folic acid tablets exerted an antiapoptotic effect, which may have occurred through the reduction of Hcy.

Hyperhomocysteinemia can induce the occurrence of oxidative stress, endothelial dysfunction, and apoptosis, which is the important pathogenesis of CIN. Enalapril maleate and folic acid tablets are a new compound preparation containing both enalapril and folic acid that can inhibit angiotensin II synthesis while reducing serum Hcy to exert renal vasodilatory, antioxidant, antiapoptotic, and endothelial function-promoting effects. This compound preparation will further exert renal protection and provide new ideas for the future treatment of CIN in clinical practice.

Several potential concerns and limitations need to be mentioned here. First, the sample size used in this study is too small. CIN model animals can not completely simulate CIN patients in clinical practice. Second, we did not attempt to measure inflammation, apoptosis protein markers, or angiotensin II levels in CIN rats due to financial constraints. However, the potential protective effects of enalapril maleate and folic acid tablets on inflammation in CIN need to be determined. Third, we did not design different doses of enalapril maleate and folic acid tablets for the treatment of CIN. CIN is still an unresolved problem in clinical practice $[41,42]$, and further animal and clinical studies are needed to determine the efficacy and safety of enalapril maleate and folic acid tablets in CIN prevention and treatment.

\section{Conclusions}

Enalapril maleate and folic acid tablets attenuated renal injury in a CIN rat model by exerting renal vasodilatory, antioxidation, antiapoptotic, and endothelial function-promoting effects. Our findings show that enalapril maleate and folic acid tablets could successfully treat CIN.

\section{Conflicts of Interest}

The authors have no conflicts of interest to declare.

\section{Authors' Contributions}

Jiantong Hou and Gaoliang Yan contributed equally to this work.

\section{Acknowledgments}

This study was supported by a grant from the National Natural Science Foundation of China to Chengchun Tang (81370225).

\section{References}

[1] F. Stacul, A. J. van der Molen, P. Reimer et al., "Contrast induced nephropathy: updated ESUR Contrast Media Safety Committee guidelines," European Radiology, vol. 21, no. 12, pp. 2527-2541, 2011.

[2] P. Aguiar-Souto, G. Ferrante, F. Del Furia, P. Barlis, R. Khurana, and C. Di Mario, "Frequency and predictors of contrastinduced nephropathy after angioplasty for chronic total occlusions," International Journal of Cardiology, vol. 139, no. 1, pp. 6874, 2010.

[3] J. Golshahi, H. Nasri, and M. Gharipour, "Contrast-induced nephropathy; a literature review," Journal of Nephropathology, vol. 3, no. 2, pp. 51-56, 2014.

[4] T. Senoo, M. Motohiro, H. Kamihata et al., "Contrast-induced nephropathy in patients undergoing emergency percutaneous coronary intervention for acute coronary syndrome," American Journal of Cardiology, vol. 105, no. 5, pp. 624-628, 2010.

[5] A. Kurtul, M. Duran, M. Yarlioglues et al., "Association between $\mathrm{N}$-terminal pro-brain natriuretic peptide levels and contrastinduced nephropathy in patients undergoing percutaneous coronary intervention for acute coronary syndrome," Clinical Cardiology, vol. 37, no. 8, pp. 485-492, 2014.

[6] C. S. Rihal, S. C. Textor, D. E. Grill et al., "Incidence and prognostic importance of acute renal failure after percutaneous coronary intervention," Circulation, vol. 105, no. 19, pp. 2259$2264,2002$.

[7] S. Demirkol, S. Balta, U. Kucuk, M. Karaman, H. O. Kucuk, and O. Kurt, "Neutrophil-to-lymphocyte ratio may predict contrastinduced nephropathy," Angiology, vol. 65, no. 1, pp. 57-58, 2014.

[8] P. A. McCullough, "Contrast-induced acute kidney injury," Journal of the American College of Cardiology, vol. 51, no. 15, pp. 1419-1428, 2008.

[9] M. Maeder, M. Klein, T. Fehr, and H. Rickli, "Contrast nephropathy: review focusing on prevention," Journal of the American College of Cardiology, vol. 44, no. 9, pp. 1763-1771, 2004.

[10] Q. Zhao, J. Yin, Z. Lu et al., "Sulodexide Protects ContrastInduced Nephropathy in Sprague-Dawley Rats," Cellular Physiology and Biochemistry, vol. 40, no. 3-4, pp. 621-632, 2016.

[11] D. Faeh, A. Chiolero, and F. Paccaud, "Homocysteine as a risk factor for cardiovascular disease: should we (still) worry about it?” Swiss Medical Weekly, vol. 136, no. 47-48, pp. 745-756, 2006.

[12] G. J. Hankey and J. W. Eikelboom, "Homocysteine and vascular disease," The Lancet, vol. 354, no. 9176, pp. 407-413, 1999.

[13] J. Malinowska, J. Kolodziejczyk, and B. Olas, "The disturbance of hemostasis induced by hyperhomocysteinemia; the role of antioxidants," Acta Biochimica Polonica, vol. 59, no. 2, pp. 185194, 2012.

[14] N. Yilmaz, "Relationship between paraoxonase and homocysteine: Crossroads of oxidative diseases," Archives of Medical Science, vol. 8, no. 1, pp. 138-153, 2012.

[15] F. Dong, X. Zhang, S.-Y. Li et al., "Possible involvement of NADPH oxidase and JNK in homocysteine-induced oxidative stress and apoptosis in human umbilical vein endothelial cells," Cardiovascular Toxicology, vol. 5, no. 1, pp. 9-20, 2005.

[16] Y. Huo, L. Liu, X. Li et al., "Pharmacology and clinical evaluation of enalapril maleate and folic acid tablet," Chinese Journal of New Drugs, vol. 19, no. 18, pp. 1633-1636, 2010. 
[17] G. Dangas, I. Iakovou, E. Nikolsky et al., "Contrast-induced nephropathy after percutaneous coronary interventions in relation to chronic kidney disease and hemodynamic variables," American Journal of Cardiology, vol. 95, no. 1, pp. 13-19, 2005.

[18] A. Ahmad, S. Mondello, R. Di Paola et al., "Protective effect of apocynin, a NADPH-oxidase inhibitor, against contrastinduced nephropathy in the diabetic rats: A comparison with n-acetylcysteine," European Journal of Pharmacology, vol. 674, no. 2-3, pp. 397-406, 2012.

[19] R. Rear, R. M. Bell, and D. J. Hausenloy, "Contrast-induced nephropathy following angiography and cardiac interventions," Heart, vol. 102, no. 8, pp. 638-648, 2016.

[20] A. Kagan and D. Sheikh-Hamad, "Contrast-induced kidney injury: Focus on modifiable risk factors and prophylactic strategies," Clinical Cardiology, vol. 33, no. 2, pp. 62-66, 2010.

[21] E. Seeliger, B. Flemming, T. Wronski et al., "Viscosity of contrast media perturbs renal hemodynamics," Journal of the American Society of Nephrology, vol. 18, no. 11, pp. 2912-2920, 2007.

[22] P. A. McCullough, J. P. Choi, G. A. Feghali et al., "ContrastInduced Acute Kidney Injury," Journal of the American College of Cardiology, vol. 68, no. 13, pp. 1465-1473, 2016.

[23] U. C. Brewster and M. A. Perazella, "The renin-angiotensinaldosterone system and the kidney: effects on kidney disease," American Journal of Medicine, vol. 116, no. 4, pp. 263-272, 2004.

[24] Z. Zhao, G. Liao, Q. Zhou, D. Lv, H. Holthfer, and H. Zou, "Sulforaphane Attenuates Contrast-Induced Nephropathy in Rats via Nrf2/HO-1 Pathway," Oxidative Medicine and Cellular Longevity, vol. 2016, Article ID 9825623, 2016.

[25] G. Wolf, "Angiotensin II as a mediator of tubulointerstitial injury," Nephrology Dialysis Transplantation, vol. 15, no. 6, pp. 61-63, 2000.

[26] F. C. Luft, "Proinflammatory effects of angiotensin II and endothelin: Targets for progression of cardiovascular and renal diseases," Current Opinion in Nephrology and Hypertension, vol. 11, no. 1, pp. 59-66, 2002.

[27] F. Yi and P.-L. Li, "Mechanisms of homocysteine-induced glomerular injury and sclerosis," American Journal of Nephrology, vol. 28, no. 2, pp. 254-264, 2008.

[28] G. Yan, W. Kong, D. Wang et al., "Impact of plasma homocysteinemia on contrast-induced nephropathy after percutaneous coronary intervention in patients with coronary syndrome," Zhonghua Xin Xue Guan Bing Za Zhi, vol. 44, no. 1, pp. 32-37, 2016.

[29] A. F. Perna, D. Ingrosso, E. Satta, C. Lombardi, F. Acanfora, and N. G. De Santo, "Homocysteine metabolism in renal failure," Current Opinion in Clinical Nutrition \& Metabolic Care, vol. 7, no. 1, pp. 53-57, 2004.

[30] X. He, L. Li, H. Tan, J. Chen, and Y. Zhou, "Atorvastatin attenuates contrast-induced nephropathy by modulating inflammatory responses through the regulation of JNK/p38/Hsp27 expression," Journal of Pharmacological Sciences, vol. 131, no. 1, pp. 18-27, 2016.

[31] L. Brattstrom and D. E. Wilcken, "Homocysteine and cardiovascular disease: Cause or effect?" American Journal of Clinical Nutrition, vol. 72, no. 2, pp. 315-323, 2000.

[32] C.-G. Zou and R. Banerjee, "Homocysteine and redox signaling," Antioxidants \& Redox Signaling, vol. 7, no. 5-6, pp. 547-559, 2005.

[33] D. E. Handy, Y. Zhang, and J. Loscalzo, "Homocysteine downregulates cellular glutathione peroxidase (GPx1) by decreasing translation," The Journal of Biological Chemistry, vol. 280, no. 16, pp. 15518-15525, 2005.
[34] J.-S. Kil, S.-O. Jeong, H.-T. Chung, and H.-O. Pae, "Piceatannol attenuates homocysteine-induced endoplasmic reticulum stress and endothelial cell damage via heme oxygenase-1 expression," Amino Acids, vol. 49, no. 4, pp. 735-745, 2017.

[35] V. S. Mujumdar, G. M. Aru, and S. C. Tyagi, "Induction of oxidative stress by homocyst(e)ine impairs endothelial function," Journal of Cellular Biochemistry, vol. 82, no. 3, pp. 491-500, 2001.

[36] J.-X. Chen and A. Stinnett, "Ang-1 gene therapy inhibits hypoxia-inducible Factor- $1 \alpha$ (HIF-1 $\alpha$ )-prolyl-4-hydroxylase-2, stabilizes HIF- $1 \alpha$ expression, and normalizes immature vasculature in db/db mice," Diabetes, vol. 57, no. 12, pp. 3335-3343, 2008.

[37] N. Weiss, "Mechanisms of increased vascular oxidant stress in hyperhomocysteinemia and its impact on endothelial function," Current Drug Metabolism, vol. 6, no. 1, pp. 27-36, 2005.

[38] H.-C. Hsu, J.-F. Chiou, Y.-H. Wang et al., "Folate deficiency triggers an oxidative-nitrosative stress-mediated apoptotic cell death and impedes insulin biosynthesis in RINm5F pancreatic islet $\beta$-cells: Relevant to the pathogenesis of diabetes," PLoS ONE, vol. 8, no. 11, Article ID e77931, 2013.

[39] S. S. Moselhy and S. H. Demerdash, "Plasma homocysteine and oxidative stress in cardiovascular disease," Disease Markers, vol. 19, no. 1, pp. 27-31, 2004.

[40] O. Postea, F. Krotz, A. Henger, C. Keller, and N. Weiss, "Stereospecific and redox-sensitive increase in monocyte adhesion to endothelial cells by homocysteine," Arteriosclerosis, Thrombosis, and Vascular Biology, vol. 26, no. 3, pp. 508-513, 2006.

[41] O. Altunoren, M. Balli, N. Eren et al., "Is Nebivolol Really Effective in Preventing Contrast Induced Nephropathy?" Kidney and Blood Pressure Research, vol. 40, no. 5, pp. 533-541, 2015.

[42] F. Wang, G. Zhang, T. Xing et al., "Renalase contributes to the renal protection of delayed ischaemic preconditioning via the regulation of hypoxia-inducible factor-1 $\alpha$," Journal of Cellular and Molecular Medicine, vol. 19, no. 6, pp. 1400-1409, 2015. 


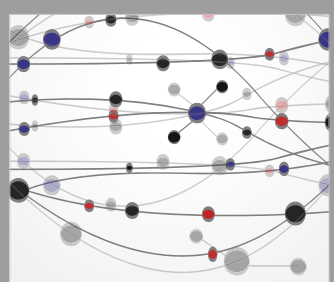

The Scientific World Journal
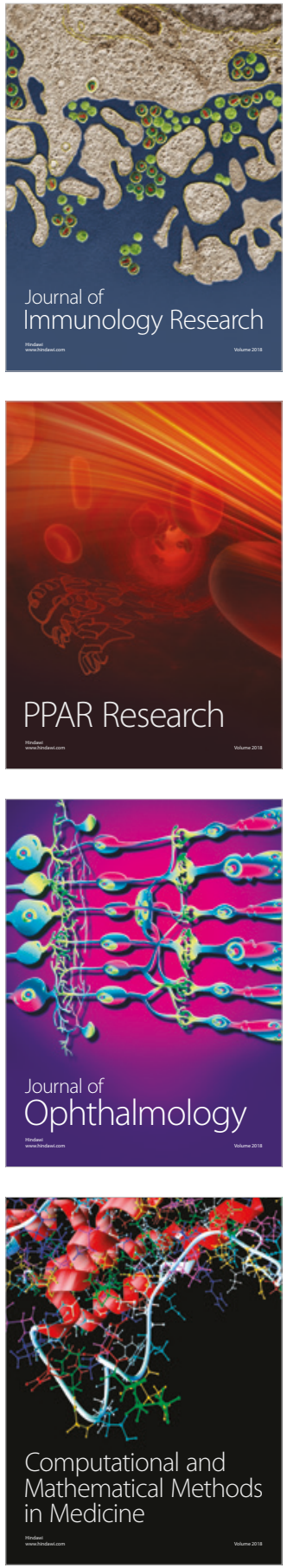

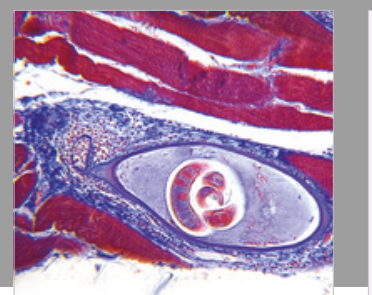

Gastroenterology Research and Practice

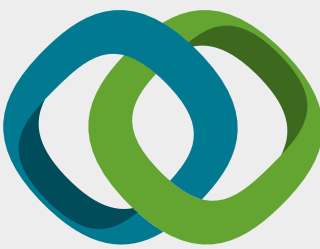

\section{Hindawi}

Submit your manuscripts at

www.hindawi.com
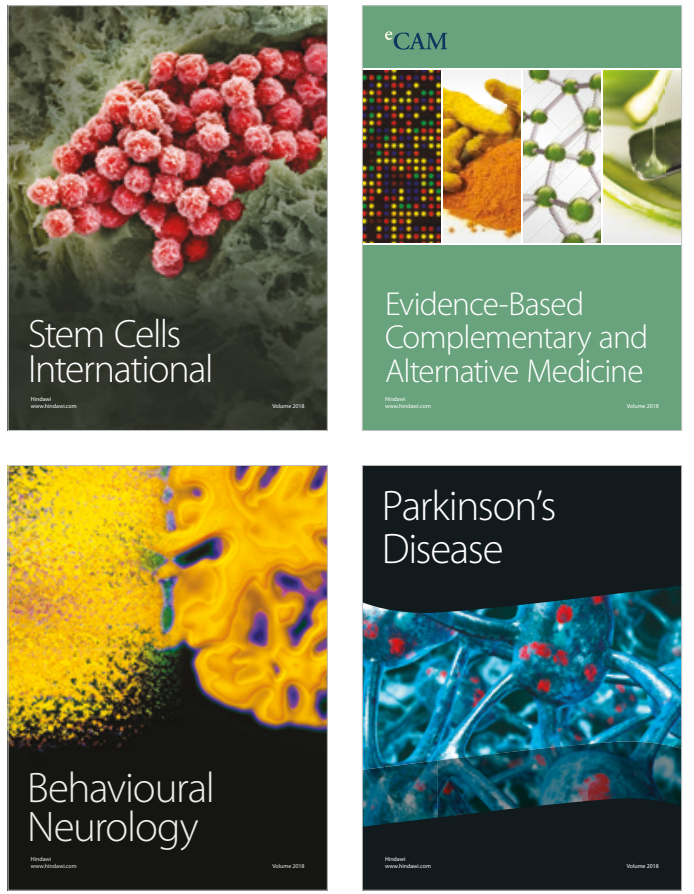

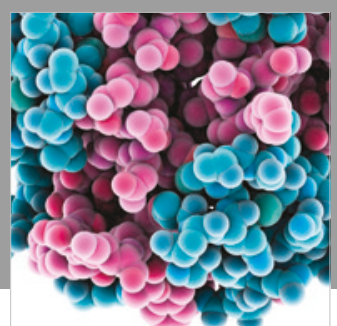

ournal of

Diabetes Research

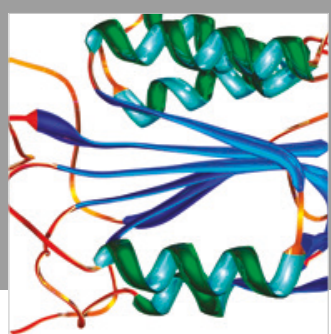

Disease Markers
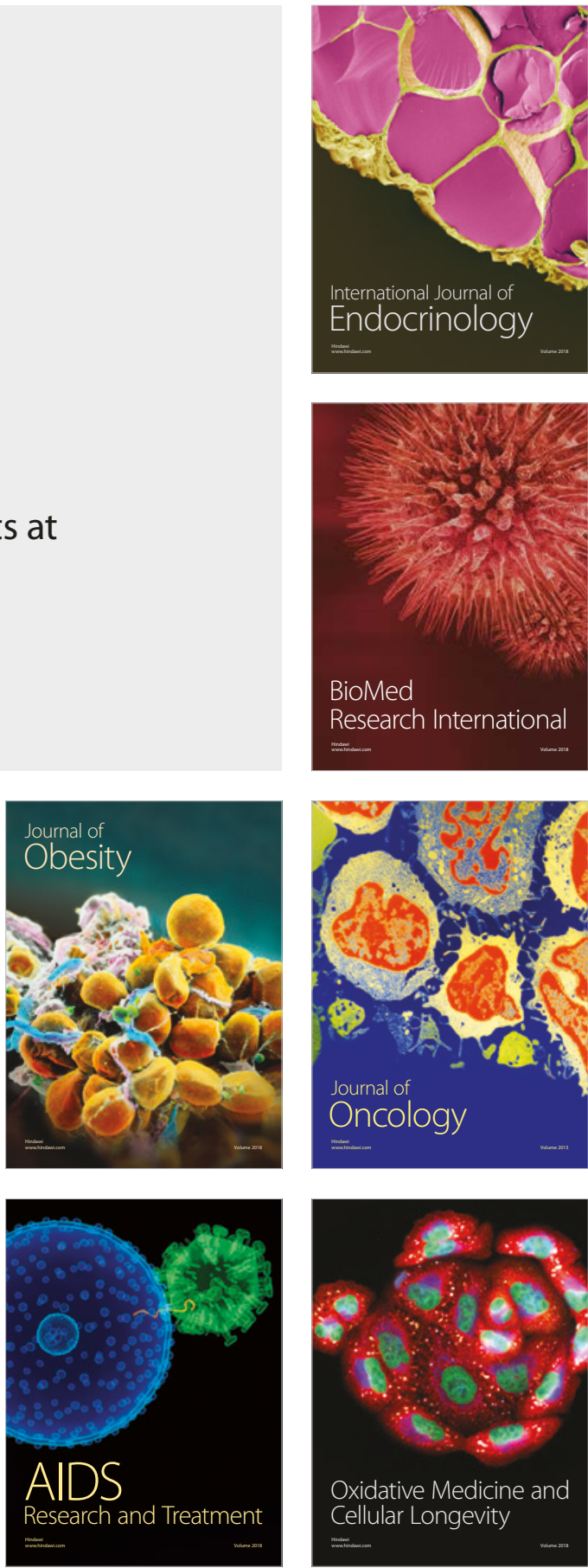